\title{
Diskussion zum Vortrag von Stefan Huster
}

\author{
Leitung: Christian STARCK
}

STARCK:

Vielen Dank für den Vortrag. Schulische Erziehungsziele sind eigentlich das einzige Beispiel, in dem wir direkte erzieherische Einflussnahme durch Gesetzgebung haben. Das andere waren ja mehr indirekte Möglichkeiten, erzieherisch zu wirken. Mir ist, als Sie jetzt mit dem zweiten Teil begonnen haben, der Gedanke gekommen, man könnte doch in der Schule auch eine Gesundheitserziehung betreiben. Herr Diederichsen hat heute schon darüber gesprochen, dass viele Eltern nicht in der Lage sind, ihre Kinder vernünftig zu erziehen. Ich weiß von einer Nichte von mir, die in Bayern in die Schule gegangen ist, dass dort Gesundheitserziehung und Ernährungswissenschaft, wenn ich das so nennen darf, als Fach angeboten wurde, und zwar als Wahlfach. Als Wahlfach, damit die Eltern sich nicht ärgern, wenn die Kinder im Unterricht gesagt bekommen, wie sie eigentlich zu Hause richtig ernährt werden müssten. Ich will die Diskussion nur eröffnen und darf jetzt fragen, wer sich dazu meldet. Zum Verfahren Folgendes: Wir haben für diese Diskussion eine halbe Stunde Zeit, danach findet eine Generaldiskussion statt. Ich würde sie ganz gern richtig abgrenzen; wir wollen uns jetzt ausschließlich zum Vortrag von Herrn Huster äußern. Herr Heun bitte.

\section{HEUN:}

Ich habe zwei Fragen, Herr Huster. Die erste ist: Sie haben sich gegen das Gleichordnungsmodell gewandt und für das Abschichtungsmodell plädiert. Da wäre zunächst einmal die Frage, wie das eigentlich dann grundrechtsdogmatisch zu fassen ist. Ich hätte jetzt sozusagen als Interpretationsvorschlag zu sagen, das ist dann eine systematische Schutzbereichsabgrenzung - oder wie würden Sie das verstehen? Ich habe mich allerdings dann nur gefragt: Kommt da wirklich so viel anderes heraus? Denn wenn wir es über die Schutzbereichsabgrenzung machen, müssen wir ja auch sagen: Was ist der Erziehungsauftrag, wie gestaltet der sich, hält der sich in den Grenzen, und das müssen wir dann wieder abgrenzen zum Elternrecht. Das heißt im Grunde genommen, wir nehmen keine Abwägung vor, sondern machen dasselbe, nur unter dem anderen Titel ,Schutzbereichsabgrenzung“. Also liegt da wirklich ein großer Unterschied oder wo würden Sie dann den Unterschied sehen? Sowohl was die Argumentation und den Argumentationsaufwand angeht als auch was das Ergebnis angeht. Das wäre der erste Punkt. Der zweite Punkt ist: Würden Sie sagen, so würde ich Sie jetzt eigentlich verstehen, dass Paternalismus des Staates verboten ist? Ist das eine 
notwendige und zwingende Konsequenz? Also, wenn man das sagt, provozieren Sie damit nicht, wenn man das mit Autonomieförderung begründet, genauso eine Heuchelei wie bei dem Nichtraucherschutz? Kommen wir nicht auch in dieselben Probleme, wenn wir mit Ihrer Beobachterperspektive argumentieren und sagen: „Es kommt dann auf soziale Schichten an“? Haben wir dann nicht das Problem, dass wir vielleicht eigentlich sagen müssten: „Na ja, also wenn das ein soziales Schichtenproblem ist, dann verbieten wir das wir für die Armen und die Reichen dürfen weiter rauchen"? Bringen Sie sich da nicht möglicherweise in Schwierigkeiten, wenn Sie ganz radikal den Paternalismus untersagen?

\section{STARCK:}

Wir nehmen wieder drei Wortmeldungen zusammen, zunächst Herr Rückert.

\section{RÜCKERT:}

Sie haben vage Formeln eingangs als Problem hingestellt. Aber ich glaube, es ist nicht viel mehr Schärfe herausgekommen bei der Abschichtung, so dass man sich doch fragt, gibt es nicht Bereiche, in denen die Schule etwa doch etwas strenger sein darf, also ganz allgemein? Und ich denke, als Juristen können wir doch eigentlich sagen: Was rechtlich anerkannte, wesentliche Werte sind im geltenden Recht, das muss doch wirklich hart vermittelt werden dürfen. Also, da hätte ich große Sympathie, einen bestimmten Bildungsbereich, nämlich den Rechtsbildungsbereich, zu den Grundfragen unseres Zusammenlebens, die anerkannt sind - und das geht auch in die Ethik rein, weil ja zum Teil diese anerkannten Grundfragen auch ethische Fragen berühren -, relativ streng durchzuführen. Neutralität kein Problem hier. Zweiter Punkt wäre: „Differenzierte Persönlichkeit“ finde ich sehr schön und auch vollkommen richtig, aber zu schwach. Ich glaube, ein wichtiger Gesichtspunkt ist diese schon etwas spöttisch auch betrachtete Autonomieförderung. Nur dass ich das nicht Autonomieförderung nennen würde, sondern etwas zurückhaltender. Es geht um die emanzipatorische Erziehung, also Erziehung dazu, dass man sich selber entfalten kann, nur um die Voraussetzungen der Autonomie und nicht um die Förderung der Autonomie das ganze Leben hindurch. So ungefähr würde ich den Unterschied sehen. Es gibt eine Reihe von Rechtsnormen, die das auch festhalten, z.B. die Unveräußerlichkeit, die die Franzosen 1789 in die Déclaration geschrieben haben, die bei uns nicht so deutlich im Grundgesetz steht, aber auch in Art. 1 und 2 GG gemeint ist: keine Selbstveräußerung in die Sklaverei. Es gibt das Verbot des Lebenszeitvertrages im Dienstvertragsrecht, $\mathbb{S} 624$ BGB. Es gibt das Verbot der finanziellen Selbstknebelung im BGB mit $₫ 311$, der inzwischen etwas verdorben ist in der Reform von 2002, aber immer noch existiert. Und ich denke, das sind gute Fallgruppenanhaltspunkte, um zu sehen, wo Schluss ist und wo Autonomie geför- 
dert werden muss bzw. die Voraussetzungen festgehalten werden müssen. Und da sehe ich eine Abgrenzungslinie, die deutlicher sein könnte, wie ich hoffe.

StARCK:

Herr Schroeder bitte.

SCHROEDER:

Sie haben in Ihrem Abschichtungsmodell gesagt, dass neutrale Erziehungsziele zulässig seien, außer wenn sie Betroffene in Gewissensnot stellen. Meiner Meinung nach legen sich diese beiden Kriterien gegenseitig lahm. Die Neutralität ist ein objektives Kriterium, Gewissensnot ein subjektives. Wir wissen, dass - sagen wir mal - bestimmte religiöse Vorstellungen, scheinbar neutrale Gebote plötzlich zu einer Gewissensnot führen. Denken wir an das Kopftuchtragen. Wenn man diese Gewissensnot als Hinderungsgrund anerkennt, was bleibt dann von den neutralen Zielen noch übrig? Ein zweiter Einwand: Sie haben mit großer Überzeugungskraft gesagt, in einem liberalen Staat müsse jeder das Recht haben, seine Gesundheit zu ruinieren. Meiner Meinung nach kollidiert das aber mit dem Gebot der sozialen Fürsorge durch den Staat, d.h. also der Krankenpflege. Wir wissen, dass die Krankheitskosten heute durch die technischen Möglichkeiten enorm gestiegen sind. Ich würde Ihnen also nur Recht geben, wenn der Betroffene vorher eine ausreichende Versicherung abgeschlossen hat, damit der Staat und die Gemeinschaft nicht für solche Exzesse aufkommen müssen.

\section{Huster:}

Zunächst zu Ihrer Vorbemerkung zur Gesundheitserziehung in der Schule. Ich meine, das mag in vielerlei Hinsicht sinnvoll sein, wir müssen aber auch aufpassen, dass wir die Schule nicht überfordern. Bei jedem gesellschaftlichen Problem sagt einer, also jetzt z.B. in der Finanzmarktkrise: Können wir nicht in der Schule den Schülern irgendwie ökonomische Zusammenhänge vermitteln? Und bei der Gesundheit muss man dann halt Gesundheitserziehung machen. Aber die Schule kann, glaube ich, auch nicht jedes Problem lösen. Außerdem, wenn man an dieser Stelle ansetzt, da finde ich es in Deutschland viel seltsamer, dass wir z.B. nicht in der Lage sind, eine vernünftige Ernährung in der Schule überhaupt anzubieten. Also ich weiß noch, als ich zur Schule gegangen bin, da waren ganz viele Fahrschüler. Die sind mittags zur Pommesbude gegangen, weil die Schule nichts angeboten hat. Eine gesundheitsdienliche Ausgestaltung der Lebenswelt vorzunehmen, könnte da doch sehr viel sinnvoller sein, als ein spezielles Fach wie Gesundheitserziehung einzuführen.

$\mathrm{Zu}$ der ersten Frage von Herrn Heun: Man kann sich natürlich überlegen: Kommt jetzt bei dem Abschichtungsmodell eigentlich etwas anderes raus als bei dem Gleichordnungsmodell? Die Frage ist deshalb schwierig zu beantwor- 
ten, weil bei diesem Abwägungs- und Gleichordnungsmodell des Bundesverfassungsgerichts die Vorgaben so vage sind und Abwägungen ja immer relativ beliebig sind, so dass man immer zu irgendeinem vernünftigen Ergebnis kommen kann. Ich meine, es geht mir zunächst einfach nur darum, die ganze Situation konsistenter $\mathrm{zu}$ strukturieren und $\mathrm{zu}$ beschreiben. $\mathrm{Ob}$ das im Ergebnis dann wirklich noch einmal etwas anderes ist, wie gesagt, das hängt im Wesentlichen davon ab, zu welchen Ergebnissen die herrschende Meinung aufgrund ihres Modells kommt.

Ist der Paternalismus schlechthin verboten? In der philosophischen Diskussion gibt es diese Unterscheidung zwischen schwachem und starkem Paternalismus. Die Grenze würde ich da sehen, wo jemand tatsächlich in vollem Bewusstsein seiner Geisteskräfte sagt: „Das ist das Leben, das ich führen will“, er dadurch auch keinen anderen in irgendeiner Weise beeinträchtigt und der Staat dann doch sagt: „Nein, das tust du nicht, weil ich besser weiß als du, was das richtige Leben ist". Das ist als Argumentationsfigur tatsächlich in einem freiheitlichen Verfassungsstaat unzulässig. Ich glaube aber, dass die Fälle in der Realität anders gelagert sind.

Führt nicht die Beobachterperspektive und die Auffassung, dass das alles sehr schichtenspezifisch ist, dazu, dass man dann auch schichtenspezifisch an die Sache herangehen müsste? Ja, Sie haben schon Recht, das ist ein Problem, das auch in unserer Public-Health-Diskussion immer mal wieder diskutiert wird, und natürlich kann das dazu führen, dass die Oberschicht der Unterschicht erklärt, wie sie ihr Leben führen soll. Und so ist das ja auch lange Zeit gelaufen. Das ist eine Gefahr, die das Gemeinwesen auch bei diesen Dingen reflektieren muss.

Zur Frage, ob nicht die rechtlich anerkannten Werte jedenfalls unproblematische Erziehungsziele in der öffentlichen Schule sind. Da würde ich Ihnen grundsätzlich sofort zustimmen, wobei ich zwei Einschränkungen machen möchte. Eigentlich müsste man es dann reduzieren auf die verfassungsrechtlich anerkannten Werte, weil vieles im einfachen Recht ja auch z.B. politisch sehr im Streit ist. Es gibt z.B. auch einen Kölner Kollegen, Herrn SchмiтTKammLER, der immer gesagt hat: „Die Schule darf nichts anderes machen, als die Verfassungswerte zu vermitteln." Das ist aber dann ein sehr unvollständiges Erziehungsprogramm, weil in der Verfassung über viele Dinge, die für die Entwicklung eines jungen Menschen doch wichtig sind, einfach nichts steht. Da die Verfassung zunächst einmal auf politische Entscheidungen und auf politische Prinzipien konzentriert ist, glaube ich, das reicht einfach nicht, um Menschen, die bis zu 13 Jahre zur Schule gehen, irgendwie ein vernünftiges Programm anzubieten. Mir persönlich gefällt der Begriff „,emanzipatorische Erziehung“ sehr gut. Der hat natürlich auch so seine Schwierigkeiten und Schlagseiten. Wenn Sie sich, was ja auch in der juristischen Diskussion eine große Rolle gespielt 
hat, die berühmten hessischen Rahmenrichtlinien ansehen, wo ja auch viel von Emanzipation usw. die Rede war - das fanden ja auch nicht alle so gut. Und Emanzipation hat natürlich auch immer so ein bisschen den Geruch von „Wir wollen die Kinder gegenüber den Bindungen, die sie mitbringen, erst einmal ein Stück weit entfremden oder diese relativieren." In dem Sinne stößt der Begriff natürlich bei den Erziehungsberechtigten auf Widerstand, weil diese versuchen, gerade diese Bindungen zu stärken. Das ist eine schwierige Gratwanderung an der Stelle.

Zu Herrn Schroeder: Relativiert die Möglichkeit, in Fällen der Gewissensnot Ausnahmen vorzusehen, nicht das objektive Kriterium, das Neutralitätsgebot? Im Grundsatz würde ich sagen, ist es natürlich immer so in einer Verfassungsordnung, die auf individuelle Gewissensnöte Rücksicht nimmt. Sie haben immer das Problem, dass dadurch allgemeine Rechts- und Verhaltenspflichten relativiert werden können. Das ist schon richtig. Worauf ich nur hinauswollte, ist: Es ist etwas anderes, zu sagen, im individuellen Fall, unter engen Voraussetzungen bin ich bereit - und natürlich dann auch immer verbunden mit einer Abwägung -, eine Befreiung vorzunehmen, als wenn ich von vornherein das gesamte Erziehungsprogramm unter den Vorbehalt der Zustimmung durch die Eltern stelle. Das scheinen mir doch zwei unterschiedliche Herangehensweisen zu sein.

Dann die Frage, ob das Recht, sein Leben zu ruinieren, nicht der sozialen Fürsorge durch den Staat widerspricht, und ob man dann nicht den Abschluss einer Zusatzversicherung verlangen müsste. Das kann man natürlich machen. In einem freiheitlichen Gemeinwesen ist es nicht systemfremd, den Einzelnen auch an den Auswirkungen seiner Lebensweise für andere teilhaben zu lassen. Nur: Ich habe mich ja in den letzten drei, vier Jahren mit dem Gesundheitswesen beschäftigt und wenn Sie sich da mal die Literatur anschauen zum Thema „Eigenverantwortung als Priorisierungs- oder Rationierungskriterium der Gesundheitsversorgung", da gibt es ganz viele Probleme - praktisch kommen Sie damit nicht sehr weit. Die Sportverletzung, die jemand sich selber zugefügt hat: Wollen Sie ihm das auferlegen oder wollen Sie sagen: „Wir wollen doch, dass die Leute sich bewegen"? Das ist alles ganz schwierig. Und deswegen glaube ich, in der Operationalisierbarkeit wird dieses Kriterium nicht sehr weiterhelfen, so ordnungspolitisch einleuchtend das in vielerlei Hinsicht natürlich ist.

STARCK:

So, jetzt kommen drei weitere Wortmeldungen: Herr Wißmann, Herr Link und Herr Alexy. 
WISSMANN:

Vielen Dank. Herr Huster, ich stehe zunächst einmal vollkommen auf Ihrer Seite, dass Sie hier stark machen, wir müssen, wenn wir vom erziehenden Gesetz und den Einwirkungen auf den Bürger sprechen, den Schüler in den Blick nehmen, denn eine Erziehungsdiktatur, Herr Alexy, ist ja eben nicht nur möglich, wenn Sie Erwachsene erziehen. Pointiert gesagt: Die ideale Erziehungsdiktatur muss den Erwachsenen gar nicht mehr erziehen, denn sie hat das Kind so erzogen, dass z.B. das BGB lupenrein auf dem Stand von 1900 erhalten bleiben könnte, aber trotzdem nicht individuell genutzt würde. Deswegen glaube ich tatsächlich, dass verfassungsrechtlich der Blick auf den Bürger, der noch nicht volljährig ist, eine ganz wichtige Ausgangsbedingung für unsere gesamte Diskussion ist. Zwei kritische Anmerkungen: Sie haben für ein Bereichsscheidungsmodell plädiert und meiner Meinung nach dann das Bundesverfassungsgericht pejorativ für etwas in Anspruch genommen, das es so nicht vertritt. Ich jedenfalls lese das nicht so, dass es hier um Einheitsvoraussetzungen oder Einheitsmodelle geht, dem man differenzierte Modelle entgegensetzen müsste. Wäre das so, würde ich Ihnen Recht geben. Ich glaube aber, das Bundesverfassungsgericht will eigentlich auf eine prozedural verschränkte Erarbeitung von Lösungskonzepten, die auch Verschiedenheit aushalten, hinaus. Das wäre jedenfalls meine Gegenanalyse der Rechtsprechung, und etwas polemisch gesagt: Diese Bereichsscheidung hatten wir ja in Deutschland bis 1949 mit den getrennten Erziehungsbereichen von Schule und Familie und es ist, denke ich, viel komplexer, dass man es ineinander verschränkt. Ganz kurz noch zu Ihrem Modell der Begründungs- und Wirkungsneutralität: Das ist natürlich faszinierend, weil es uns anbietet, Komplexität zu reduzieren. Ich frage Sie aber noch einmal: Was schließt denn das Modell der Begründungsneutralität aus, wenn es nicht mal verpflichtenden Ethikunterricht ausschließt? Das wäre das eine. Und die Wirkungsneutralität: Hat die nicht eine enorme jakobinische Gefahr, wenn man einfach aus dem Blick nimmt, dass allgemeine Regelungen extrem unterschiedlich wirken können und dass das die Bürger extrem unterschiedlich auch betreffen kann? Deswegen wäre mein letzter Punkt: Sollten wir nicht die politische Entscheidung auch in Erziehungsfragen bewusst stärker machen: Die Gemeinschaft entscheidet darüber, was sie für richtig hält, durch das Gesetz?

STARCK:

Herr Link bitte.

LINK:

Ich will es kurz machen. Ich bin, Herr Huster, mit Ihnen völlig einverstanden; erstens damit, dass in diesen Gesundheitsfragen der Drittschutz eine Heuchelei ist, jedenfalls in seinen extremen Regelungen. Ein gewisses Unbehagen habe 
ich wie Herr Heun auch bezüglich der schichtenspezifischen Selbstgefährdungen. Ist das nicht auch ein Ausdruck des Paternalismus, wenn man Selbstgefährdungen danach beurteilt, ob sie schichtenspezifisch sind? Dann müsste man Drachenfliegen - sicher nicht schichtenspezifisch, jedenfalls nicht unterschichtenspezifisch, weil es viel zu teuer ist - doch wohl auch verbieten. Oder - ich bin auch Raucher - wird eine Schicht dann in Geiselhaft genommen für andere Schichten? Geht hier nicht die Beurteilungskompetenz des Staates über schichtenspezifisches Verhalten zu weit in Ihrem Modell?

\section{STARCK:}

Herr Alexy bitte.

Alexy:

Ich habe eine Bemerkung zum Verhältnis von Gleichordnungsmodell und Abschichtungsmodell. Ein Argument zur Kritik des Gleichordnungsmodells von Ihnen, Herr Huster, war, dass den subjektiven Vorstellungen der Eltern zu viel Raum gegeben werde. Nun hängt aber die Beurteilung der Eingriffsintensität doch keinesfalls allein von den subjektiven Vorstellungen der Eltern ab. Das ist vielmehr nur ein Faktor, der in eine objektive Beurteilung einzustellen ist. Das ist mein erster Punkt. Mein zweiter Punkt ist vielleicht wichtiger: Sie haben Verhältnismäßigkeit und Neutralität nebeneinander gestellt. Meine Frage lautet nun: Muss man die Neutralität nicht doch in den Verhältnismäßigkeitsgrundsatz integrieren? Etwa nach der Regel: Je neutraler ein Eingriff ist, desto weniger intensiv ist er.

STARCK:

Vielen Dank, jetzt gibt Herr Huster Antworten.

Huster:

Ja, bei der ersten Frage von Herrn Wißmann will ich zunächst nur für die Anregung danken. Ich gebe zu, dass ich vielleicht eine etwas grobschlächtige, zugespitzte Rekonstruktion der Rechtsprechung des Bundesverfassungsgerichts vorgenommen habe. Das kann man auch differenzierter machen, aber da ging es mir vor allem darum, überhaupt ein Gegenmodell zu präsentieren. Der zweite Punkt war: Was verbietet die Begründungsneutralität, wenn sie den verpflichtenden Ethikunterricht zulässt? Ich würde sagen, sie lässt z.B. einen Ethikunterricht dann eben nicht zu, in dem ganz dezidiert z.B. religionsfeindlich oder zugunsten einer bestimmten oder gegen eine andere Religion Stellung bezogen wird. Bei der Sache mit der Wirkungsneutralität sehe ich weniger eine jakobinische Gefahr, sondern ich gebe zu, ich neige da zu einem gewissen Etatismus. Und ich glaube, dass wir die Frage der Schwere der Auswirkung und deren Zumutbarkeit in einem komplexen Zusammenspiel von politischem Prozess und 
Anpassung an die jeweiligen Lebensformen entscheiden müssen. Typischerweise müssen die Mehrheitsgesellschaft und die Minderheitskultur da in irgendeiner Weise aufeinander zugehen, sich aneinander abarbeiten. Mein wichtiger Punkt ist nur, dass das Verfassungsrecht das nicht vorwegnehmen kann. Das müssen wir durch einen politischen Prozess in Gang setzen.

Zu Herrn Link: Warum ist die Sache mit der schichtenspezifischen Ausrichtung des Gesundheitsverhaltens so wichtig? Sie ist deshalb wichtig, weil anders als beim Drachenfliegen, was eine punktuelle Entscheidung ist, wo man dem Einzelnen wirklich sagen kann: „Das hast du dir jetzt überlegt, am Sonntagnachmittag Drachenfliegen zu gehen, das hättest du auch sein lassen können“, das bei den lebensstilbezogenen Dingen, wenn jemand sich falsch ernährt, sich zu wenig bewegt, zu viel trinkt, alles sehr viel schwieriger ist und mit der gesamten Lebenssituation zu tun hat. Das ist ja nicht ganz zufällig, dass Leute, die sonst schon mit ihrem Leben viele Probleme haben, auch mit ihrem Gesundheitsverhalten nicht zurechtkommen. Es ist daher ein bisschen unfair, einfach die Verantwortung immer dem Einzelnen zuzuweisen, wenn wir sehen, dass ganz viele in der gleichen Situation auch ganz entsprechende Probleme haben. Die Statistiken sind da eindeutig. Der Unterschied in der Lebenserwartung zwischen dem obersten und dem untersten Einkommensfünftel in Deutschland beträgt bei Männern 10 Jahre. Das muss man sich überlegen, 10 Jahre. Da hilft auch die medizinische Versorgung nicht, sondern das hängt sehr allgemein mit der Lebenssituation dieser Menschen zusammen.

Zur ersten Frage von Herrn Alexy. Natürlich ist die Beurteilung von Eingriffsintensitäten nicht nur an den subjektiven Vorstellungen orientiert. Aber ich würde schon sagen, wie man ja an den Fällen auch sieht, dass die Abwertung, die Aversion, das Nichtmögen von bestimmten erzieherischen Einwirkungen, wie einen koedukativen Sportunterricht, nun doch schon ganz gewaltig von den jeweiligen subjektiven Erziehungsvorstellungen abhängt und auch davon, ob der Einzelne - so beurteilen das ja auch die Verwaltungsgerichte - darlegen kann, dass ihn das in eine Gewissensnot bringt oder ob ihm das einfach nur nicht so behagt. Und das scheint mir doch stark von den subjektiven Vorstellungen abzuhängen und deswegen würde ich sagen, das ist jedenfalls ein Faktor, der eine erhebliche Rolle spielt. Dann zum Verhältnismäßigkeitsprinzip und zum Neutralitätsprinzip: Mein Modell ist, zu sagen, die Verhältnismäßigkeit schützt gegen zu intensive Eingriffe, die Neutralität schützt gegen Eingriffe aus den falschen Gründen. Und ich glaube, dass das eine sinnvolle Zuordnung ist. Man kann natürlich auch versuchen, diesen Neutralitätsgedanken z.B. in der Angemessenheitsstufe der Verhältnismäßigkeitsprüfung unterzubringen. Da spricht grundsätzlich nichts dagegen; ich glaube nur, wenn man das nebeneinanderstellt, dass das transparenter wird. Ich nenne Ihnen einen Parallelfall, wo wir das so 
ähnlich machen, nämlich bei der Meinungsfreiheit und dem Begriff der allgemeinen Gesetze, wo wir auch die Sonderrechtslehre und die Abwägungslehre nebeneinander haben. Und auch da haben wir genau die gleiche Struktur: Die Sonderrechtslehre schützt gegen Eingriffe aus den falschen Gründen, die Abwägungslehre schützt allgemein gegen zu intensive Eingriffe in die Meinungsfreiheit. Auch da könnte man sagen, besonders intensiv sind eben Eingriffe, die nicht meinungsneutral sind. Aber wir unterscheiden es dort und ich glaube, es trägt zur Transparenz bei, wenn wir zwei unterschiedliche Prüfungspunkte daraus machen.

STARCK:

Ich habe noch eine Wortmeldung, Herr Spickhoff bitte.

SPICKHOFF:

Mich interessiert noch eine Frage zum Thema der staatlichen Gesundheitserziehung. Sie schließt an die Bemerkung von Herrn Link an, Stichwort: Paternalismus und die vorgeschlagene Differenzierung nach sozialen Hintergrundfaktoren. Die Frage ist, wie weit man eigentlich bei dieser Differenzierung nach sozialen Hintergrundfaktoren gehen sollte. Herr Huster, Sie würden ja - was ich nicht kritisch sehe - paternalistisch z.B. sagen: In einschlägigen Gegenden darf man aufgrund der schichtenspezifischen Gefahr nicht rauchen. Die Frage ist dann aber, ob man dort ein Raucherlokal für Professorinnen und Professoren doch eröffnen darf. Noch weitergehend: Müsste man McDonald's abschaffen, jedenfalls in einschlägigen Gegenden, weil die dort angebotenen Lebensmittel zu einseitig sind? Läge das nicht sogar auf der Linie Ihrer Ausführungen? Könnte man noch weitergehen, und fragen, ob Kinder auf der Hauptschule zu Kinderregeluntersuchungen gezwungen werden sollen, und wie steht es mit der (Wieder-)Einführung eines Impfzwangs für bestimmte Personengruppen? Oder sollte im Falle von Eingriffen in die körperliche Integrität besser doch nicht nach Schichten differenziert werden, weil sich hier das Grundrecht auf Selbstbestimmung im Kontext der ärztlichen Aufklärung durchsetzt? Es ist kein Geheimnis, dass auch im Bereich der Patientinnen und Patienten schichtenspezifisch der Verständnishorizont durchaus uneinheitlich ist. Deshalb könnte man vertreten, dass man sich bei Patientinnen und Patienten, die einen begrenzten Empfängerhorizont haben, um eine besonders eingehende Aufklärung zu bemühen hat. Es könnte aber auch geschehen, dass solche Patienten aus solchen Gruppen oder Bevölkerungsschichten dann, wenn ihnen das höchste Risiko mitgeteilt worden ist, gebotene Eingriffe aufgrund von missverstandener Angst unterlassen. Das könnte zu der Idee verführen, dass man entsprechende Patientengruppen gar nicht oder geringer aufklärt und paternalistisch den Eingriff durchführt, was zwar ganz der überholten ärztlichen Linie entsprechen würde, aber nicht mehr 
unserem heutigen Leitbild. Im Endeffekt ist die Frage also die, wie weit man mit entsprechenden Differenzierungen gehen sollte.

STARCK:

Herr Huster, bitte jetzt Ihre letzte Antwort.

Huster:

Ich habe ja versucht anzudeuten, dass es für all diese Fragen keine Zauberformel gibt. Und man kann sich dann natürlich auch so ein bisschen darüber lustig machen und jetzt sagen: „Müssen wir nicht McDonald's in bestimmten Gegenden verbieten?"“. Ich sage Ihnen nur andersrum, dass sind Dinge, die die Industrie natürlich schon lange macht. Werbung und auch die Ansiedlung von Einrichtungen mit derartigen gesundheitsgefährdenden Produkten sind extrem schichtenspezifisch ausgeprägt. Die schauen sich natürlich genau an: „Bei wem kann ich mit dem Zeug landen, welche Menschen in welcher Lebenssituation haben gegen unsere Werbeeinflüsterungen überhaupt keinen Widerstand mehr anzubieten?" Und deswegen scheint mir die Idee, dass das Gemeinwesen auf diese Strategien der Ernährungsindustrie dadurch reagiert, dass es dann an bestimmten Stellen sagt: „Nein, Fernsehwerbung verbieten wir jetzt“, nicht so abwegig zu sein. Im Übrigen müsste man sich die Fälle im Einzelnen ansehen. Ich glaube, die Sache mit der ärztlichen Aufklärung ist noch mal eine ganz andere Geschichte.

STARCK:

Vielen Dank. 